$\Rightarrow$ NEUROIMMUNOLOGY

\section{A brain drain}

Immunologists have long believed that the central nervous system (CNS) lacks lymphatic vessels and have struggled to understand how lymphocytes access and exit the brain. Louveau et al. now describe the existence of functional lymphatic vessels that run parallel to the dural sinuses of the meninges. The discovery of these lymphatics is likely to have far-reaching consequences for our understanding of immune responses in the CNS.

The authors set out to characterize the pathways that recirculating immune cells use to survey the meningeal spaces. Immunostaining of mouse brain meninges showed that T cells and MHC class IIexpressing cells were concentrated in close proximity to the dural sinuses (which drain blood from the CNS) but were typically not found within these blood vessels. Instead, these immune cells aligned in a linear fashion in vessel structures adjacent to the sinuses. Surprisingly, these vessel structures stained positive for the expression of lymphatic vessel endothelial hyaluronic acid receptor 1 (LYVE1) and other classical markers of lymphatic endothelial cells, suggesting that they were meningeal lymphatics.

The meningeal lymphatics showed features typical of initial (as opposed to collecting) afferent lymphatics and extended from above the eyes and olfactory bulb to sites adjacent to the dural sinuses. Experiments using intracerebral injection of tracer dyes showed that the meningeal lymphatic vessels are functional and can drain cerebrospinal fluid (CSF), albeit at a slower rate than the adjacent blood vessels. In addition, immunostaining experiments suggested that B cells, T cells and CD11 $\mathrm{c}^{+}$myeloid cells traffic through the meningeal lymphatics.

It has been proposed that CSF constituents drain to the cervical lymph nodes via lymphatics in the nasal mucosa. However, the authors found that meningeal lymphatics, rather than nasal lymphatics, are the primary route through which CSF drains to deep cervical lymph nodes (DCLNs). Indeed, resection of the DCLNs in naive mice led to an accumulation of $\mathrm{T}$ cells in the meninges. Furthermore, ligation of the lymphatic vessels that drain into the DCLNs increased the diameter of the meningeal lymphatics in a manner similar to lymphoedema.

The authors propose that the newly discovered meningeal lymphatics could be important for the second stage of draining interstitial fluid from the brain parenchyma, after it has initially been drained into the CSF via the glymphatic system (see further reading). They also suggest that malfunction of these vessels may lie at the heart of various neurological disorders.

Yvonne Bordon

ORIGINAL RESEARCH PAPER Louveau, A. et al. Structural and functional features of central nervous system lymphatic vessels. Nature http://dx.doi.org/10.1038/nature14432 (2015) FURTHER READING Yuhas, D. How the brain cleans itself. Nature http://dx.doi.org/10.1038/ nature.2012.11216 (2012) 


\section{ONLINE ONLY}

\section{Subject categories:}

Biological sciences / Immunology / Lymphatic system /

Lymphatic vessels

[URI /631/250/1617/2069]

Biological sciences / Immunology / Neuroimmunology

[URI /631/250/371]

Web summary:

Lymphatic vessels have been identified in the central nervous system. 